\title{
AN EXTENSION OF WRIGHT'S 3/2-THEOREM FOR THE KPP-FISHER DELAYED EQUATION
}

\author{
KAREL HASIK AND SERGEI TROFIMCHUK
}

(Communicated by Yingfei Yi)

\begin{abstract}
We present a short proof of the following natural extension of Wright's famous 3/2-stability theorem: the conditions $\tau \leq 3 / 2, c \geq 2$ imply the presence of the positive traveling fronts (not necessarily monotone) $u=$ $\phi(x \cdot \nu+c t),|\nu|=1$, in the delayed KPP-Fisher equation $u_{t}(t, x)=\Delta u(t, x)+$ $u(t, x)(1-u(t-\tau, x)), u \geq 0, x \in \mathbb{R}^{m}$.
\end{abstract}

\section{INTRODUCTION AND MAIN RESULT}

The delayed KPP-Fisher (i.e. Kolmogorov-Petrovskii-Piskunov-Fisher) equation

$$
u_{t}(t, x)=\Delta u(t, x)+u(t, x)(1-u(t-\tau, x)), u \geq 0, x \in \mathbb{R}^{m}, \tau \geq 0,
$$

is one of the most conspicuous examples of delayed reaction-diffusion equations. During the past decade, this model and the non-local version of the KPP-Fisher equation

$$
u_{t}(t, x)=\Delta u(t, x)+u(t, x)\left(1-\int_{\mathbb{R}^{n}} K(y) u(t, x-y) d y\right), \int_{\mathbb{R}^{n}} K(s) d s=1,
$$

have been intensively studied by many authors; see e.g. 2, 6, 8, 10, 12, 18,

One of the key topics related to equations (1.1), (1.2) concerns the existence and further properties of smooth positive traveling front solutions $u(x, t)=\phi(\nu \cdot x+c t)$, $|\nu|=1$ for (1.1). It is supposed that $c>0$ and that the profile $\phi$ satisfies the boundary conditions $\phi(-\infty)=0, \phi(+\infty)=1$. Just a few years ago, not much was known about the conditions guaranteeing the existence of these wavefronts in (1.1). Several existence results having a rather partial character were provided in [18] (for any $c>2$ and $\tau \in[0, \tau(c)]$ with sufficiently small $\tau(c)$ ) and in [6.7] (for any $\tau \leq 3 / 2$ and $c \geq c(\tau)$ with sufficiently large $c(\tau))$. In this respect, significant progress was achieved only very recently when the existence and uniqueness problems for (1.1), (1.2) were completely solved for the case of monotone profiles [2, 4, 5, 8, 10, 12, However, the monotonicity of $\phi$ is a rather restrictive assumption: it is clear that traveling fronts of (1.1), (1.2) that oscillate around 1 at $+\infty$ (hence, non-monotone ones) comprise the largest part of the set of all wavefront solutions [1,2, 10, 16]. In this note, by establishing an 'almost optimal criterion' for the presence of oscillating

Received by the editors February 5, 2013 and, in revised form, March 7, 2014.

2010 Mathematics Subject Classification. Primary 34K10, 35K57; Secondary 92D25.

Key words and phrases. KPP-Fisher equation, Wright's 3/2-theorem, delay.

This research was realized within the framework of the OPVK program, project CZ.1.07/2.300/20.0002.

The second author was also partially supported by FONDECYT (Chile), project 1110309, and by CONICYT (Chile) through PBCT program ACT-56. 
fronts in equation (1.1), we achieve an essential improvement of the existence results from $[7,8,12,18$. Still, the complete solution of the mentioned problem remains quite a challenging project which is directly connected to Wright's long-standing global stability conjecture [11,17.

Let us explain the last comment in more detail. Indeed, looking for a wave solution of (1.1) in the slightly modified form $u(t, x)=\psi(\sqrt{\epsilon} x+t), \epsilon=1 / c^{2}$, $\psi(s)=\phi(c s)$, we find that

$$
\epsilon \psi^{\prime \prime}(t)-\psi^{\prime}(t)+\psi(t)(1-\psi(t-\tau))=0, t \in \mathbb{R}
$$

In the limit case, $\epsilon=0$, equation (1.3) is called the Hutchinson equation, and it was conjectured by E.M. Wright [17] that the steady state $\psi=1$ of (1.3) with $\epsilon=0$ is globally stable in the domain of all positive solutions $\psi>0$ if and only if $\tau \leq \pi / 2$. A weaker version of Wright's conjecture can also be considered: the Hutchinson equation has a positive heteroclinic connection (i.e., a traveling front type solution) if and only if $\tau \leq \pi / 2$. Both conjectures are supported by the 'very difficult theorem of Wright' (the quoted phrase is from Jack Hale's book [9, p. 64]) proved in [17]: If $\tau \leq 3 / 2$, then the positive equilibrium of (1.3) with $\epsilon=0$ is globally stable in the domain of positive solutions. Remarkably, as was shown in [6,7] by means of the Hale-Lin approach, Wright's 3/2-theorem can be extended to (1.3) with $\epsilon>0$ in the following way: equation (1.3) has a positive heteroclinic connection for each positive fixed $\tau \leq 3 / 2$ if $\epsilon>0$ is sufficiently small. The main result of this work shows that the smallness condition on $\epsilon$ (i.e. the requirement that the propagation speed $c$ has to be sufficiently large) can be avoided and that the full analog of Wright's theorem holds for (1.1):

Theorem 1.1. Assume that $c \geq 2$ and $\tau \in[0,3 / 2]$. Then the delayed KPP-Fisher reaction-diffusion equation (1.1) has at least one positive traveling front solution.

It is well known that the inequality $c \geq 2$ is mandatory for the existence of positive wavefronts $[2,8,10$. We also believe that, similarly to the monotone fronts [4, 5, 8, 10, there is a unique (up to a translation) oscillating front for each fixed $c$.

Theorem 1.1 strongly supports the next generalisation of the weak Wright conjecture [10]: equation (1.1) has at least one positive traveling front $u=\phi(\nu \cdot x+c t)$, $|\nu|=1$, if and only if $c \geq 2$ and the equation $\lambda^{2}-c \lambda-e^{-\lambda c \tau}=0$ has a unique root $\lambda$ with positive real part. In particular, this means that the maximal possible improvement of the interval $[0,3 / 2]$ in Theorem 1.1 is $[0, \pi / 2]$; see [10, Figure 1].

The starting point for the proof of Theorem 1.1 is the fact that, for each $c \geq 2$, equation (1.1) (similarly to equation (1.2); see [2]) has at least one positive wave solution $u=\phi(\nu \cdot x+c t),|\nu|=1$, satisfying the boundary conditions $\phi(-\infty)=$ $0,0<m=\liminf _{t \rightarrow+\infty} \phi(t) \leq \limsup _{t \rightarrow+\infty} \phi(t)=M<+\infty$ (i.e. a semiwavefront); see [10]. The next important fact is that each non-monotone semiwavefront profile is sine-like slowly oscillating around 1 at $+\infty[10,14,15$. In Section [3, we present several explicit analytic relations limiting the amplitude of these oscillations. At a first glance, the mentioned restrictions are generated by rather cumbersome bounding functions. Surprisingly, these functions have nice internal structures (previously analysed in [13]) that allow for their satisfactory description in Section 2, At the very end of Section 3, in order to demonstrate Theorem 1.1 we show that $\tau \leq 3 / 2$ together with $c \geq 2$ implies $m=M=1$. 


\section{Auxiliary functions}

Our approach to the proof of Theorem 1.1 requires the construction of several suitable bounding functions. These functions are necessary to relate the values of $m$ and $M$ (defined a few lines above); it is clear that their choice is by no means unique. Below, we present our auxiliary functions and prove their properties, which are later used in the proof of Theorem 1.1. First, we consider $\rho(x)=\rho(x, \tau, c):=\tau c f(w(x))$, where $f(x):=\frac{-c+\sqrt{c^{2}+4 x}}{2}, \quad w(x):=e^{-x}-1$.

Proposition 2.1 ([10]). Let $c \geq 2$. Then the real analytic function $\rho(x), x \in \mathbb{R}$, $\rho(0)=0, \rho(-\infty)=+\infty, \rho(+\infty)=-0.5 \tau c\left(-c+\sqrt{c^{2}-4}\right)<0$, is strictly decreasing (in fact, $\rho^{\prime}(x)<0, x \in \mathbb{R}$ ) and has a negative Schwarz derivative $(S \rho)(x)$ on $\mathbb{R}$ : $(S \rho)(x)=\rho^{\prime \prime \prime}(x) / \rho^{\prime}(x)-3 / 2\left(\rho^{\prime \prime}(x) / \rho^{\prime}(x)\right)^{2}<0, x \in \mathbb{R}$.

It is straightforward to see that $\rho$ is a convex function:

$$
\rho^{\prime \prime}(x)=c \tau e^{-x}\left(f^{\prime}(w(x))\right)^{3}\left(c^{2}-4+2 e^{-x}\right)>0, \quad x \in \mathbb{R} .
$$

Corollary 2.2. If $c \geq 2$, then for all $x>0$, it holds that

$$
\rho(x)>r(x):=\frac{\rho^{\prime}(0) x}{1-0.5 \rho^{\prime \prime}(0) x / \rho^{\prime}(0)}=\frac{-\tau x}{1+0.5\left(1-2 / c^{2}\right) x} .
$$

Proof. This is an immediate consequence of Proposition 2.1 and [13, Lemma 2.1].

Next, for each $c \geq 2, \tau \in(1,3 / 2]$, we consider

$$
\begin{gathered}
A_{-}(x, c, \tau)=x+\rho(x)+\frac{1}{\rho(x)} \int_{x}^{0} \rho(s) d s, \quad x \neq 0 \\
A_{+}(x, c, \tau)=x+r(x)+\frac{1}{r(x)} \int_{x}^{0} r(s) d s, \quad B(x, c, \tau):=\frac{1}{r(x)} \int_{-r(x)}^{0} r(s) d s, \quad x>0 .
\end{gathered}
$$

It is easy to see that $A_{ \pm}, B$ are continuous at $x=0$ if we set $A_{ \pm}(0, c, \tau)=$ $B(0, c, \tau)=0$. Observe also that $B(x, c, \tau)$ is strictly decreasing on $\mathbb{R}_{+}$,

$$
\begin{gathered}
A_{ \pm}^{\prime}(0, c, \tau)=\frac{1}{2}-\tau, \quad A_{ \pm}^{\prime \prime}(0, c, \tau)=\left(\tau-\frac{1}{6}\right)\left(1-\frac{2}{c^{2}}\right) \\
A_{-}(x, c, \tau)<A_{-}(x, c, 3 / 2), x<0, \tau>1 \\
A_{+}(x, c, \tau)>A_{+}(x, c, 3 / 2), x>0, \tau>1 \\
B(x, c, \tau)>B(x, c, 3 / 2), x>0, \tau>1 .
\end{gathered}
$$

Let $x_{2}>0$ be the unique positive solution of equation $-r(x)=x$. Since $\tau>1$, it holds, for a positive $x$, that $x / r(x)>-1$ if and only if $x \in\left(0, x_{2}\right)$. As was established in [13, Lemma 2.3], $A_{+}(x, c, \tau)$ is strictly decreasing in the first variable on $\left(-\infty, x_{2}\right]$. The next result has a similar proof:

Lemma 2.3. $A_{-}^{\prime}(x, c, \tau)<0$ and $\left(S A_{-}\right)(x, c, \tau)<0$ once $x / \rho(x)>-1$.

Proof. Using the convexity of $\rho$ and recalling that $-\rho^{\prime}(0)=\tau>1, \rho(0)=0$, it is easy to see that $x / \rho(x)>-1$ if and only if $x<\bar{x}_{2}$ where $\bar{x}_{2}$ is the unique positive solution of equation $-\rho(x)=x$. In consequence, $x \rho(x)+\rho^{2}(x)>0, x<\bar{x}_{2}, x \neq 0$,

$$
A_{-}^{\prime}(x, c, \tau)=\rho^{\prime}(x)\left(1-\frac{\int_{x}^{0} \rho(s) d s}{\rho^{2}(x)}\right)<\rho^{\prime}(x)\left(1+\frac{x \rho(x)}{\rho^{2}(x)}\right)<0, x<\bar{x}_{2}, x \neq 0 .
$$


We know also that $A_{-}^{\prime}(0, c, \tau)=0.5-\tau<0$. Now, integrating by parts, we obtain

$$
A_{-}(x, c, \tau)=\rho(x)+\frac{x \rho(x)+\int_{\rho(x)}^{0} v d \theta(v)}{\rho(x)}=\rho(x)+\frac{1}{\rho(x)} \int_{0}^{\rho(x)} \theta(v) d v=G(\rho(x)),
$$

where $\theta(v):=\rho^{-1}(v)$ and $G(z)=z+\int_{0}^{1} \theta(v z) d v$.

Then, by Proposition 2.1 and by the formula for the Schwarzian derivative of the composition of two functions, we obtain

$$
\left(S A_{-}\right)(x, c, \tau)=(S G)(\rho(x))\left(\rho^{\prime}(x)\right)^{2}+(S \rho)(x)<(S G)(\rho(x))\left(\rho^{\prime}(x)\right)^{2} .
$$

Thus the negativity of $S A_{-}$will follow from the inequality $(S G)(\rho(x))<0$. Since $A_{-}^{\prime}(x, c, \tau)<0$ if and only if $G^{\prime}(\rho(x))>0$, it suffices to show that $(S G)(\rho(x))<0$ when $G^{\prime}(\rho(x))>0$. Now, in view of Proposition 2.1.

$$
\theta^{\prime \prime \prime}(\rho(x))=\frac{3\left(\rho^{\prime \prime}(x)\right)^{2}-\rho^{\prime \prime \prime}(x) \rho^{\prime}(x)}{\left(\rho^{\prime}(x)\right)^{5}}=\frac{-(S \rho)(x)}{\left(\rho^{\prime}(x)\right)^{3}}+\frac{3}{2} \frac{\left(\rho^{\prime \prime}(x)\right)^{2}}{\left(\rho^{\prime}(x)\right)^{5}}<0 .
$$

Hence, $G^{\prime \prime \prime}(z)=\int_{0}^{1} v^{3} \theta^{\prime \prime \prime}(v z) d v<0, z=\rho(x)$, and therefore $(S G)(\rho(x))<$ $G^{\prime \prime \prime}(\rho(x)) / G^{\prime}(\rho(x))<0$. This completes the proof of Lemma 2.3

Next, for $c \geq 2, \tau \in(1,3 / 2]$, we will also consider the functions

$$
\begin{aligned}
& R(x, c, \tau)=\frac{A_{+}^{\prime}(0, c, \tau) x}{1-0.5 A_{+}^{\prime \prime}(0, c, \tau) x / A_{+}^{\prime}(0, c, \tau)}, \\
& D(x, c, \tau)= \begin{cases}A_{-}(x, c, \tau) & \text { if } x \leq 0, \\
A_{+}(x, c, \tau) & \text { if } x \in\left[0, x_{2}\right], \\
B(x, c, \tau) & \text { if } x \geq x_{2} .\end{cases}
\end{aligned}
$$

As the above discussion shows, $D(x, c, \tau)$ is strictly decreasing in $x \in \mathbb{R}$. From now on, we fix $\tau=3 / 2$ and set $A_{ \pm}(x, c):=A_{ \pm}(x, c, 3 / 2), B(x, c):=B(x, c, 3 / 2)$, $D(x, c):=D(x, c, 3 / 2), R(x, c):=R(x, c, 3 / 2)$. The strictly decreasing function $D(x, c)$ has the following additional nice property:

Proposition 2.4. If $c \geq 2$, then $D(x, c)>R(x, c)$ for all $x>0$.

Proof. The above inequality follows from [13, Corollary 2.7] if we take there $f^{\prime}(0)=$ $r^{\prime}(0)=-\tau=-3 / 2$. It should be observed that the definitions of functions $A, B, r, R$ in 13 are identical to the definitions of $A_{+}, B, r, R$ in this paper. The only formal difference with [13] is the presence of parameter $c$ in the expressions for the second derivatives of $A_{+}, r, R$ at 0 . However, once these derivatives are positive, the proofs in [13] do not depend on their exact values; e.g. see Lemma 2.6 from [13].

Corollary 2.5. $F(x):=A_{-}(R(x))<x$ for all $x>0$.

Proof. By Lemma 2.3. $(S F)(x)=\left(S A_{-}\right)(R(x))\left(R^{\prime}(x)\right)^{2}<0$ for all $x$ from some open neighbourhood of $[0,+\infty)$. Also $S R \equiv 0$, so that

$$
0=(S R)(0)=-R^{\prime \prime \prime}(0)-1.5\left(R^{\prime \prime}(0)\right)^{2}=-R^{\prime \prime \prime}(0)-1.5\left(A_{-}^{\prime \prime}(0)\right)^{2} .
$$

Next, we have that $F^{\prime}(0)=-R^{\prime}(0)=1, F^{\prime \prime}(0)=A_{-}^{\prime \prime}(0)\left(R^{\prime}(0)\right)^{2}+A_{-}^{\prime}(0) R^{\prime \prime}(0)=$ $0, F^{\prime \prime \prime}(0)=A_{-}^{\prime \prime \prime}(0)\left(R^{\prime}(0)\right)^{3}+3 A_{-}^{\prime \prime}(0) R^{\prime}(0) R^{\prime \prime}(0)+A_{-}^{\prime}(0) R^{\prime \prime \prime}(0)=\left(S A_{-}\right)(0)<0$. Therefore $F(x)<x$ for all small positive $x$. Now, suppose that $F(z)=z$ for some leftmost positive $z$. Then $F^{\prime}(z) \geq 1$ and therefore the function $y=F^{\prime}(x)>0$, $x \in[0, z], F^{\prime}(0)=1$, has a positive local minimum at some point $p \in(0, d)$. But then $F^{\prime \prime}(p)=0, F^{\prime \prime \prime}(p) \geq 0$, and therefore $(S F)(p) \geq 0$, a contradiction. 


\section{Bounding RElations And the CONVERGEnCE OF SEMI-WAVEFronts}

As we have mentioned in the introduction, for each $c \geq 2$, equation (1.1) has at least one positive wave solution $u=\phi(\nu \cdot x+c t),|\nu|=1$, satisfying the boundary conditions $\phi(-\infty)=0,0<\liminf _{t \rightarrow+\infty} \phi(t) \leq \limsup _{t \rightarrow+\infty} \phi(t)<+\infty$. Clearly, $\phi$ satisfies

$$
\phi^{\prime \prime}(t)-c \phi^{\prime}(t)+\phi(t)(1-\phi(t-h))=0, h:=c \tau, t \in \mathbb{R} .
$$

The change of variables $\phi(t)=e^{-x(t)}$ transforms the latter equation into

$$
x^{\prime \prime}(t)-c x^{\prime}(t)-\left(x^{\prime}(t)\right)^{2}+\left(e^{-x(t-h)}-1\right)=0, t \in \mathbb{R} .
$$

By Theorem 1.4 from [10, $x(t)$ is sine-like slowly oscillating around 0. More precisely, there exists an increasing sequence $Q_{j}, j \geq 0$, of zeros of $x(t)$ such that $x(t)<$ 0 on $\left(Q_{0}, Q_{1}\right) \cup\left(Q_{2}, Q_{3}\right) \cup \ldots$ and $x(t)>0$ on $\left(-\infty, Q_{0}\right) \cup\left(Q_{1}, Q_{2}\right) \cup\left(Q_{3}, Q_{4}\right) \cup \ldots$ Furthermore, $x(t)$ has exactly one critical point (hence, local extremum point) $T_{j}$ on each interval $\left[Q_{j}, Q_{j+1}\right]$ and $T_{j}-Q_{j}<h$ for all $j$. Hence, $y(t):=x^{\prime}(t)$ does not change its sign on the intervals $\left(T_{j}, T_{j+1}\right), j=0,1,2 \ldots$, and $y\left(T_{j}\right)=0$. Therefore $y$ solves the boundary value problem

$$
y^{\prime}=y^{2}+c y-g(t), \quad y\left(T_{j}\right)=y\left(T_{j+1}\right)=0,
$$

where $c \geq 2$ and $g(t):=w(x(t-h))$ is $C^{2}$-smooth on $\mathbb{R}$.

Lemma 3.1. For each integer $j \geq 0$, solution $y(t)$ has a unique critical point (absolute minimum point) $p_{j} \in\left[T_{2 j+1}, T_{2 j+2}\right]$, and for all $t \in\left(p_{j}, T_{2 j+2}\right)$, it holds that $y(t)>\rho(x(t-h)) / h$. Furthermore, for each non-increasing function $M=M(t)$, $t \in\left[Q_{2 j}, Q_{2 j+2}\right]$, such that $x(t) \leq M(t), t \in\left[Q_{2 j}, Q_{2 j+2}\right]$, it holds that

$$
y(t)>\rho(M(t-h)) / h, t \in\left(T_{2 j+1}, T_{2 j+2}\right) \backslash\left\{p_{j}\right\} .
$$

Similarly, for $j \geq 1$, solution $y(t)$ has a unique critical point (absolute maximum point) $q_{j} \in\left[T_{2 j}, T_{2 j+1}\right]$, and for all $t \in\left(q_{j}, T_{2 j+1}\right)$, it holds that $y(t)<\rho(x(t-h)) / h$. Furthermore, for each non-decreasing function $m=m(t), t \in\left[Q_{2 j-1}, Q_{2 j+1}\right]$, such that $x(t) \geq m(t), t \in\left[Q_{2 j-1}, Q_{2 j+1}\right]$, it holds that

$$
y(t)<\rho(m(t-h)) / h, t \in\left(T_{2 j}, T_{2 j+1}\right) \backslash\left\{q_{j}\right\} .
$$

Proof. We will prove only the first assertion of the lemma, the proof of the second statement being completely analogous. Consider the slope field for differential equation (3.3). Two zero isoclines

$$
\lambda_{1}(t)=\frac{-c-\sqrt{c^{2}+4 g(t)}}{2}<-\frac{c}{2}<\frac{-c+\sqrt{c^{2}+4 g(t)}}{2}:=\lambda_{2}(t)
$$

partition the plane $\mathbb{R}^{2}$ into three horizontal bands:

$\Pi_{1}=\left\{(t, y): y \leq \lambda_{1}(t)\right\}, \Pi_{2}=\left\{(t, y): \lambda_{1}(t) \leq y \leq \lambda_{2}(t)\right\}, \Pi_{3}=\left\{(t, y): y \geq \lambda_{2}(t)\right\}$, limited by the graphs of functions $y=\lambda_{1}(t), y=\lambda_{2}(t)$. We observe that the portions of integral curves of (3.3) belonging to the interior of domains $\Pi_{1}, \Pi_{3}$ [respectively, $\Pi_{2}$ ] are increasing [respectively, decreasing]. Since $y\left(T_{2 j+2}\right)=0$ and $g\left(T_{2 j+2}\right)=\exp \left(-x\left(T_{2 j+2}-h\right)\right)-1<0$ we find that $\left(T_{2 j+2}, 0\right) \in \operatorname{Int} \Pi_{3}$, where Int $X$ denotes the interior part of the set $X$. Similarly, $\left(T_{2 j+1}, 0\right) \in \operatorname{Int} \Pi_{2}$ while the points $T_{2 j+1}$ and $T_{2 j+2}$ are separated by a unique zero $Q_{2 j+1}+h$ of $y=\lambda_{2}(t)$ on $\left[T_{2 j+1}, T_{2 j+2}\right]$. As a consequence, the integral curve of each function $y(t)$ solving (3.3) never enters $\Pi_{1}$ and belongs to $\Pi_{2} \cup \Pi_{3}$. Moreover, it is clear that $y^{\prime}(t)>0$ 
on some maximal interval $\left(p_{j}, T_{2 j+2}\right)$ where $y\left(p_{j}\right)=\lambda_{2}\left(p_{j}\right), y^{\prime}\left(p_{j}\right)=0$. Since clearly $0 \leq y^{\prime \prime}\left(p_{j}\right)=-g^{\prime}\left(p_{j}\right)$, the point $\left(p_{j}, \lambda_{2}\left(p_{j}\right)\right)$ lies on the decreasing part of the graph $\Gamma$ of $y=\lambda_{2}(t)$ (observe that $\lambda_{2}^{\prime}(t)=g^{\prime}(t) / \sqrt{c^{2}+4 g(t)}$ ). We claim that $(t, y(t))$ does not cross $\Gamma$ again for all $t \in\left[T_{2 j+1}, p_{j}\right)$. Indeed, otherwise there would exist some $d \in\left[Q_{2 j+1}+h, p_{j}\right)$ such that $y(d)=\lambda_{2}(d)$ and therefore $y^{\prime}(d)=0$, while $\lambda_{2}^{\prime}(d)=g^{\prime}(d) / \sqrt{c^{2}+4 g(d)}<0$ since $g^{\prime}(t)=-x^{\prime}(t-h) \exp (-x(t-h))<0$, $t \in\left[Q_{2 j+1}+h, p_{j}\right), g\left(p_{j}\right) \leq 0$. This means that at the moment $t=d$ the integral curve of the solution $y=y(t)$ intersects transversally $\Gamma$, enters the domain $\Pi_{3}$ and is strictly increasing on $\left(d, p_{j}\right)$. Since $y=\lambda_{2}(t)$ is strictly decreasing on the same interval, we get a contradiction: $y\left(p_{j}\right)>\lambda_{2}\left(p_{j}\right)$.

Hence, we have the following description of the behaviour of each solution $y(t)$ to (3.3) on $\left[T_{2 j+1}, T_{2 j+2}\right]$ : there exists a point $p_{j} \in\left(T_{2 j+1}, T_{2 j+2}\right)$ such that

i) $y^{\prime}(t)>0, y(t)>\lambda_{2}(t)=\rho(x(t-h)) / h, \quad t \in\left(p_{j}, T_{2 j+2}\right]$;

ii) $y^{\prime}\left(p_{j}\right)=0, y\left(p_{j}\right)=\lambda_{2}\left(p_{j}\right)$;

iii) $y^{\prime}(t)<0, y(t)<\lambda_{2}(t), \quad t \in\left[T_{2 j+1}, p_{j}\right)$.

Finally, in order to justify (3.4), we observe that $\left[Q_{2 j}, Q_{2 j+2}\right] \supset\left[T_{2 j+1}-h, T_{2 j+2}-h\right]$, see [10], Theorem 3.4]. Therefore, since $\rho$ decreases on $\mathbb{R}$, we obtain that $\rho(x(t-$ $h)) \geq \rho(M(t-h))$ for $t \in\left[T_{2 j+1}, T_{2 j+2}\right]$. Thus the property i) implies (3.4) for all $t \in\left(p_{j}, T_{2 j+2}\right]$. In particular, $y\left(p_{j}\right) \geq \rho\left(M\left(p_{j}-h\right)\right) / h$. Since in addition, $y(t)$ is strictly decreasing on $\left[T_{2 j+1}, p_{j}\right)$ and $\rho(M(t-h))$ is non-decreasing on the same interval, we conclude that (3.4) also holds for all $t \in\left[T_{2 j+1}, p_{j}\right)$. This completes the proof of Lemma 3.1 .

Remark 3.2. For the oscillating semi-wavefront solutions $x=x(t)$ of equation (3.2), the above result improves considerably the estimates of Lemma 4.1 from [10. In order to obtain such an improvement, we have used here our knowledge of the slowly oscillating behaviour of $g(t)$ : this information was not relevant for the proof of Lemma 4.1.

Corollary 3.3. The profiles of oscillating semi-wavefronts of equation (1.1) have a unique inflection point between two consecutive extremum points.

In the next stage of our studies, we will evaluate the extremal values $V_{j}=x\left(T_{j}\right)$ for $j \geq 1$ (it follows from [10, Corollary 1] that $V_{0} \geq-c h$ ).

Lemma 3.4. Let $c \geq 2, \tau \in(1,3 / 2]$ and $x(t)=-\ln \phi(t)$ oscillate on $\left[Q_{0},+\infty\right)$. Then $V_{2 j+1} \leq A_{-}\left(V_{2 j}, c, \tau\right), j \geq 0, V_{2 j} \geq B\left(V_{2 j-1}, c, \tau\right), j \geq 1$. If, in addition, $V_{2 j-1} \leq x_{2}$, then $V_{2 j} \geq A_{+}\left(V_{2 j-1}, c, \tau\right), j \geq 1$.

Proof. As we know, $V_{1}=x\left(T_{1}\right)>0$ with $T_{1}-Q_{0}>h$ and $x^{\prime}\left(T_{1}\right)=0, x\left(Q_{1}\right)=0$, $T_{1}-Q_{1}<h$. Set $Q_{-1}=T_{-1}=-\infty$. It is clear that $x(s) \geq V_{0}$ for all $s \in\left[Q_{-1}, Q_{1}\right]$. On the other hand, due to Lemma 3.1, we know that

$$
x^{\prime}(t) \leq \max _{s \in\left[T_{0}, T_{1}\right]} x^{\prime}(s) \leq \frac{1}{h} \rho\left(\min _{s \in\left[Q_{-1}, Q_{1}\right]} x(s)\right) \leq \frac{1}{h} \rho\left(V_{0}\right), \quad t \in\left[T_{0}, T_{1}\right],
$$

and therefore

$$
x(t)=-\int_{t}^{Q_{1}} x^{\prime}(s) d s \geq-\frac{1}{h} \int_{t}^{Q_{1}} \rho\left(V_{0}\right) d s=\frac{\rho\left(V_{0}\right)}{h}\left(t-Q_{1}\right)=\tilde{m}(t), \quad t \in\left[T_{0}, Q_{1}\right] .
$$

In particular, $x\left(T_{0}\right)=V_{0} \geq \tilde{m}\left(T_{0}\right)$ and therefore the equation $\tilde{m}(t)=V_{0}$ has a root $t_{1} \in\left[T_{0}, Q_{1}\right]$. Since $V_{0}<0$, we know from the first lines of the proof of Lemma 2.3 
that $t_{1}-Q_{1}=h V_{0} / \rho\left(V_{0}\right)>-h$. Consider now the non-decreasing function

$$
m(t)= \begin{cases}\tilde{m}(t) & \text { if } t \in\left[t_{1}, Q_{1}\right] \subset\left(Q_{1}-h, Q_{1}\right], \\ V_{0} & \text { if } t \leq t_{1} .\end{cases}
$$

It is clear that $x(t) \geq m(t)$ for all $t \in\left[Q_{-1}, Q_{1}\right]$. Therefore, by Lemma 3.1 .

$$
V_{1}=\int_{Q_{1}}^{T_{1}} x^{\prime}(s) d s \leq \frac{1}{h} \int_{Q_{1}}^{T_{1}} \rho(m(s-h)) d s \leq \frac{1}{h} \int_{Q_{1}-h}^{Q_{1}} \rho(m(s)) d s=A_{-}\left(V_{0}, c, \tau\right) .
$$

Next, consider $V_{2}=x\left(T_{2}\right)<0$ : we have $x^{\prime}(t)<0$ on $\left(T_{1}, T_{2}\right), x^{\prime}\left(T_{2}\right)=0, x\left(Q_{2}\right)=0$ and $T_{2}-Q_{2}<h$. By Lemma 3.1 and Corollary 2.2

$$
x^{\prime}(t) \geq \min _{s \in\left[T_{1}, T_{2}\right]} x^{\prime}(s) \geq \frac{1}{h} \rho\left(\max _{s \in\left[Q_{0}, Q_{2}\right]} x(s)\right) \geq \frac{\rho\left(V_{1}\right)}{h}>\frac{r\left(V_{1}\right)}{h}, \quad t \in\left[T_{1}, T_{2}\right],
$$

and therefore

$$
x(t)=-\int_{t}^{Q_{2}} x^{\prime}(s) d s<\frac{r\left(V_{1}\right)}{h}\left(t-Q_{2}\right)=\tilde{M}(t), \quad t \in\left[T_{1}, Q_{2}\right) .
$$

Since $\tilde{M}(t)$ is decreasing on $\left[Q_{0}, Q_{2}\right], \tilde{M}\left(T_{1}\right)>x\left(T_{1}\right)=V_{1}$ and $\max _{t \in\left[Q_{0}, Q_{2}\right]} x(t)=$ $x\left(T_{1}\right)$, we have that $x(t)<\tilde{M}(t)$ for $t \in\left(Q_{0}, Q_{2}\right]$. Then Lemma 3.1 yields

$$
V_{2}=\int_{Q_{2}}^{T_{2}} x^{\prime}(s) d s \geq \frac{1}{h} \int_{Q_{2}-h}^{Q_{2}} \rho(\tilde{M}(s)) d s>\frac{1}{h} \int_{Q_{2}-h}^{Q_{2}} r(\tilde{M}(s)) d s=B\left(V_{1}, c\right) .
$$

Suppose now that $V_{1} \leq x_{2}$. Let $t=t_{2}$ solve the equation $h V_{1}=r\left(V_{1}\right)\left(t-Q_{2}\right)$; then $t_{2}-Q_{2}=h V_{1} / r\left(V_{1}\right) \geq-h$ (see the comments following the definition of $x_{2}$ ). Consider the non-increasing function

$$
M(t)= \begin{cases}\tilde{M}(t) & \text { if } t \in\left[t_{2}, Q_{2}\right] \subset\left[Q_{2}-h, Q_{2}\right], \\ V_{1} & \text { if } t \leq t_{2}\end{cases}
$$

it is clear that $x(t) \leq M(t)$ for all $t \in\left[Q_{0}, Q_{2}\right]$. By applying Lemma 3.1 and Corollary 2.2, we obtain

$$
V_{2}=\int_{Q_{2}}^{T_{2}} x^{\prime}(s) d s>\frac{1}{h} \int_{Q_{2}-h}^{Q_{2}} \rho(M(s)) d s>\frac{1}{h} \int_{Q_{2}-h}^{Q_{2}} r(M(s)) d s=A_{+}\left(V_{1}, c\right) .
$$

Finally, we can repeat the above arguments to obtain similar estimates for all $j>2$. This completes the proof of Lemma 3.4

We are now in a position to finalise the proof of Theorem 1.1. Consider the following finite limits:

$$
0 \geq m_{*}=\liminf _{j \rightarrow+\infty} V_{j}=\liminf _{t \rightarrow+\infty} x(t), \quad 0 \leq M_{*}=\limsup _{j \rightarrow+\infty} V_{j}=\limsup _{t \rightarrow+\infty} x(t) .
$$

From Lemmas 2.3 and 3.4 we deduce that $M_{*} \leq A_{-}\left(m_{*}, c, \tau\right)$ and $m_{*} \geq D\left(M_{*}, c, \tau\right)$. Clearly, Theorem 1.1 will be proved if we show that $\tau \leq 1.5$ yields $M_{*}=0$. Since this implication was already proved for $\tau \leq 1$ in [10, Theorem 1.7], we may assume that $\tau>1$. Suppose that $M_{*}>0, \tau \in(1,3 / 2]$. However, then, due to inequalities (2.1) and Proposition 2.4, $M_{*} \leq A_{-}\left(m_{*}, c, \tau\right)<A_{-}\left(m_{*}, c\right)$ and $m_{*} \geq D\left(M_{*}, c, \tau\right)>D\left(M_{*}, c\right)>R\left(M_{*}\right)$. Therefore we have $M_{*}<A_{-}\left(R\left(M_{*}\right)\right)$. However, by Corollary 2.5. $A_{-}\left(R\left(M_{*}\right)\right)<M_{*}$, a contradiction. Hence, $M_{*}=0$ and the proof of Theorem 1.1 is completed. 


\section{REFERENCES}

[1] P. Ashwin, M. V. Bartuccelli, T. J. Bridges, and S. A. Gourley, Travelling fronts for the KPP equation with spatio-temporal delay, Z. Angew. Math. Phys. 53 (2002), no. 1, 103-122, DOI 10.1007/s00033-002-8145-8. MR:1889183 (2002m:35097)

[2] Henri Berestycki, Grégoire Nadin, Benoit Perthame, and Lenya Ryzhik, The non-local FisherKPP equation: travelling waves and steady states, Nonlinearity 22 (2009), no. 12, 2813-2844, DOI 10.1088/0951-7715/22/12/002. MR 2557449 (2010j:35438)

[3] Arnaud Ducrot and Grégoire Nadin, Asymptotic behaviour of travelling waves for the delayed Fisher-KPP equation, J. Differential Equations 256 (2014), no. 9, 3115-3140, DOI 10.1016/j.jde.2014.01.033. MR3171769

[4] Jian Fang and Jianhong Wu, Monotone traveling waves for delayed Lotka-Volterra competition systems, Discrete Contin. Dyn. Syst. 32 (2012), no. 9, 3043-3058, DOI 10.3934/dcds.2012.32.3043. MR.2912070

[5] Jian Fang and Xiao-Qiang Zhao, Monotone wavefronts of the nonlocal Fisher-KPP equation, Nonlinearity 24 (2011), no. 11, 3043-3054, DOI 10.1088/0951-7715/24/11/002. MR2844826 (2012k:35287)

[6] Teresa Faria, Wenzhang Huang, and Jianhong Wu, Travelling waves for delayed reactiondiffusion equations with global response, Proc. R. Soc. Lond. Ser. A Math. Phys. Eng. Sci. 462 (2006), no. 2065, 229-261, DOI 10.1098/rspa.2005.1554. MR2189262(2006i:35182)

[7] Teresa Faria and Sergei Trofimchuk, Positive travelling fronts for reaction-diffusion systems with distributed delay, Nonlinearity 23 (2010), no. 10, 2457-2481, DOI 10.1088/09517715/23/10/006. MR2683776(2011g:35192)

[8] Adrian Gomez and Sergei Trofimchuk, Monotone traveling wavefronts of the KPPFisher delayed equation, J. Differential Equations 250 (2011), no. 4, 1767-1787, DOI 10.1016/j.jde.2010.11.011. MR2763555(2012c:35213)

[9] Jack K. Hale, Asymptotic behavior of dissipative systems, Mathematical Surveys and Monographs, vol. 25, American Mathematical Society, Providence, RI, 1988. MR.941371 (89g:58059)

[10] Karel Hasik and Sergei Trofimchuk, Slowly oscillating wavefronts of the KPP-Fisher delayed equation, Discrete Contin. Dyn. Syst. 34 (2014), no. 9, 3511-3533, DOI 10.3934/dcds.2014.34.3511. MR3190991

[11] Balázs Bánhelyi, Tibor Csendes, Tibor Krisztin, and Arnold Neumaier, Global attractivity of the zero solution for Wright's equation, SIAM J. Appl. Dyn. Syst. 13 (2014), no. 1, 537-563, DOI 10.1137/120904226. MR 3183042

[12] Man Kam Kwong and Chunhua Ou, Existence and nonexistence of monotone traveling waves for the delayed Fisher equation, J. Differential Equations 249 (2010), no. 3, 728-745, DOI 10.1016/j.jde.2010.04.017. MR.2646048 (2012c:35237)

[13] Eduardo Liz, Manuel Pinto, Gonzalo Robledo, Sergei Trofimchuk, and Victor Tkachenko, Wright type delay differential equations with negative Schwarzian, Discrete Contin. Dyn. Syst. 9 (2003), no. 2, 309-321. MR1952376 (2004a:34135)

[14] John Mallet-Paret and George R. Sell, Systems of differential delay equations: Floquet multipliers and discrete Lyapunov functions, J. Differential Equations 125 (1996), no. 2, 385-440, DOI 10.1006/jdeq.1996.0036. MR1378762(97a:34193a)

[15] John Mallet-Paret and George R. Sell, The Poincaré-Bendixson theorem for monotone cyclic feedback systems with delay, J. Differential Equations 125 (1996), no. 2, 441-489, DOI 10.1006/jdeq.1996.0037. MR.1378763 (97a:34193b)

[16] Grégoire Nadin, Benoît Perthame, and Min Tang, Can a traveling wave connect two unstable states? The case of the nonlocal Fisher equation (English, with English and French summaries), C. R. Math. Acad. Sci. Paris 349 (2011), no. 9-10, 553-557, DOI 10.1016/j.crma.2011.03.008. MR2802923 (2012d:35193) 
[17] E. M. Wright, A non-linear difference-differential equation, J. Reine Angew. Math. 194 (1955), 66-87. MR0072363(17,272b)

[18] Jianhong Wu and Xingfu Zou, Traveling wave fronts of reaction-diffusion systems with delay, J. Dynam. Differential Equations 13 (2001), no. 3, 651-687, DOI 10.1023/A:1016690424892. MR.1845097 (2003a:35114)

Mathematical Institute, Silesian University, 74601 Opava, Czech Republic

E-mail address: Karel.Hasik@math.slu.cz

Instituto de Matemática y Física, Universidad de Talca, Casilla 747, Talca, Chile

E-mail address: trofimch@inst-mat.utalca.cl 